ISAHP 2005, Honolulu, Hawaii, July 8-10, 2005

\title{
ANALYSIS OF NETWORK ECONOMY BY ANP/DNP ${ }^{1}$
}

\author{
Petr Fiala \\ Department of Econometrics, University of Economics \\ Praha, 130 67, Czech Republic \\ pfiala@vse.cz
}

Keywords: network economy, positive feedback, complementarity, Analytic Network Process, Dynamic Network Process

Summary: The network economy is a term for today's global relationship among economic subjects characterized by massive connectivity. The central act of the new era is to connect everything to everything in deep web networks at many levels of mutually interdependent relations, where resources and activities are shared, markets are enlarged and costs and risk are reduced. Network systems contain both positive and negative feedbacks. A variety of feedback processes create complex system behavior. For the whole network seems to be very appropriate Analytic Network Process (ANP) approach. The ANP method makes possible to deal systematically with all kinds of dependence and feedback in the system. Dynamic models try to reflect changes in real or simulated time and take into account that the network model components are constantly evolving. Dynamic models use concepts of state variables, flows, and feedback processes. Dynamic Network process (DNP) as an extension of ANP can deal with time dependent priorities in network economy.

\section{Introduction}

Network economy drives and is driven by dramatic acceleration in technological innovation, in information and communication technologies especially. New technologies provide a permanent feedback that enables activity modifications and quick responses and therefore fundamentally change business models. The analysis of possible effects of network economy is an opportune topic for challenging scientific research. Business process modeling is a using of models and methods for understanding and change of the processes in relation to information systems of firms. The relation between business models and information systems becomes more and more tighter. Using technology to connect ever more closely with network users creates "connectivity paradox" in which new realms of opportunity coexist with new forms of risk.

Applications of the network problems are found in transportation, telecommunications, network reliability settings, finance, knowledge and other applications. The unifying concept of global networks with associated methodologies allows to explore the interactions among such networks as transportation networks, telecommunication networks, as well as financial networks. The basic problems and their solutions can be combined for more complex situations with multiple decision-makers and multiple criteria. The main aim of the paper is to propose a methodological framework for analyzing network economy. Many specific factors of the network economy can be modeled and analyzed by ANP/DNP methods. Some specific features as network externalities, complementarities, supplier selection problem are analyzed by ANP/DNP methods and combined in a general model of network economy.

\footnotetext{
${ }^{1}$ The paper is partially supported by the Grant Agency of Czech Republic (grant 402/05/0148).
} 


\section{Network economy}

The network economy (Economides, 1996, Kelly, 1998, Shapiro, Varian, 1999, Shy, 2001) is a term for today's global relationship among economic subjects characterized by massive connectivity. The central act of the new era is to connect everything to everything in deep web networks at many levels of mutually interdependent relations, where resources and activities are shared, markets are enlarged and costs of risk are reduced.

Network industries play a crucial role in modern life. Today network systems provide the infrastructure and foundation for the functioning of societies and economies. They come in many forms and include physical networks such as: transportation and logistical networks, communication networks, energy networks, as well as more abstract networks comprising: economic and financial networks, environmental networks, social, and knowledge networks. Many important non-network industries share many essential economic features with network industries. These non-network industries are characterized by strong complementary relations.

The reality of today's networks includes features:

- large-scale nature and complexity,

- increasing congestion,

- complementarity,

- externalities,

- switching costs,

- alternative behaviors of users of the networks,

- interactions between the networks themselves.

Many of today's networks are characterized by both a large-scale nature and complexity of the network topology. Congestion is playing an increasing role in not only transportation networks but also in telecommunication networks. The crucial relationship in networks is the complementarity between the pieces of the network. Complementarity turns to be a crucial factor in the markets for information goods. Networks exhibit positive externalities. The value of a unit of the good increases with the expected number of units to be sold. Cost of switching to a different service or adopting a new technology are significant. There are various types of these costs as contracts, training and learning, data conversion, search costs etc. The decisions made by the users of the networks, in turn, affect not only the users themselves but others, as well, in terms of profits and costs, timeliness of deliveries, the quality of the environment, etc. The behavior of the users of the networks themselves may be non-cooperative. One of the principal facets of the network economy is the interaction among the networks themselves.

The aim of the paper is to develop models and computational framework to address new opportunities in network connections of different units. Significant factors for network models comprise:

- $\quad$ network structure,

- dynamic environment,

- uncertainty environment,

- multiple decision units with multiple goals,

- system optimization versus local optimization

The factors of network connectedness, permanent feedback, coordination and cooperation possibilities bringing synergistic effects. These aspects can be analyzed by modeling and optimization techniques, such as Dynamic Network Process. The instrument is suitable to model the network economy appropriately with an accent on main features o network economy.

Network systems contain both positive and negative feedbacks. These processes are different and vary in strength. A variety of feedback processes create complex system behavior. Dynamic models try to reflect changes in real or simulated time and take into account that the network model components are constantly evolving. 


\section{The ANP/DNP methods}

The Analytic Hierarchy Process (AHP) is the method for setting priorities (Saaty, 1996). A priority scale based on reference is the AHP way to standardize non-unique scales in order to combine multiple performance measures. The AHP derives ratio scale priorities by making paired comparisons of elements on a common hierarchy level by using a 1 to 9 scale of absolute numbers. The absolute number from the scale is an approximation to the ratio $\mathrm{w}_{\mathrm{j}} / \mathrm{w}_{\mathrm{k}}$ and then is possible to derive values of $\mathrm{w}_{\mathrm{j}}$ and $\mathrm{w}_{\mathrm{k}}$. The AHP method uses the general model for synthesis of the performance measures in the hierarchical structure.

$$
u_{i}=\sum_{j=1}^{n} v_{j} w_{j k} .
$$

The Analytic Network Process (ANP) is the method (Saaty, 2001) that makes it possible to deal systematically with all kinds of dependence and feedback in the performance system. The well-known AHP theory is a special case of the Analytic Network Process that can be very useful for incorporating linkages in the performance system.

The structure of the ANP model is described by clusters of elements connected by their dependence on one another. A cluster groups elements (success factors, managerial measures, process drivers, business units) that share a set of attributes. At least one element in each of these clusters is connected to some element in another cluster. These connections indicate the flow of influence between the elements ( see Figure 1).

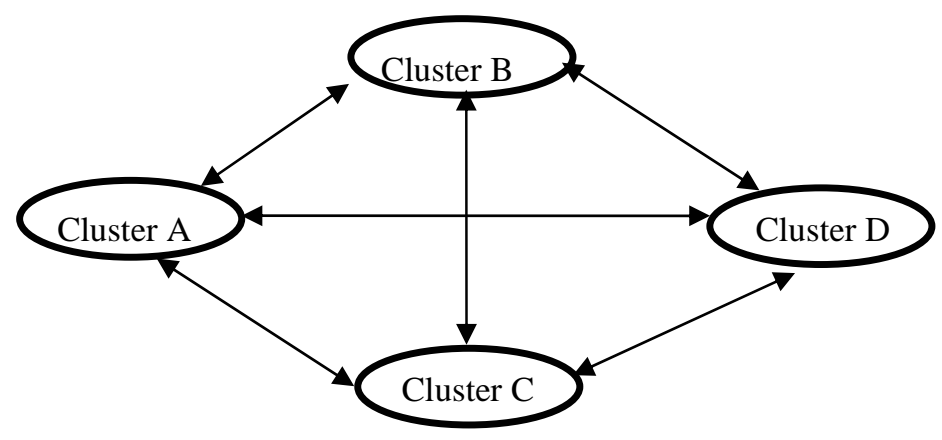

Figure 1 Flows of influence between the elements

The clusters in the supplier selection problem can be suppliers, producers, customers, and evaluating criteria also. The connections among members of supply chain networks are material, financial and information flows.

Paired comparisons are inputs for computing a global performance of network systems. A supermatrix is a matrix of all elements by all elements. The weights from the paired comparisons are placed in the appropriate column of the supermatrix. The sum of each column corresponds to the number of comparison sets. The weights in the column corresponding to the cluster are multiplied by the weight of the cluster. Each column of the weighted supermatrix sums to one and the matrix is column stochastic. Its powers can stabilize after some iterations to limited supermatrix. The columns of each block of the matrix are identical and we can read off the global priority of units.

The AHP and ANP have been static but for today's world analyzing is very important time dependent decision making. The DHP/DNP (Dynamic Hierarchy Process/ Dynamic Network Process) methods were introduced (Saaty, 2003). There are two ways to study dynamic decisions: structural, by including scenarios, and functional by explicitly involving time in the judgment process. For the functional dynamics there are analytic or numerical solutions. The basic idea with the numerical approach is to obtain the time dependent principal eigenvector by simulation. 


\section{Network effects}

Networks exhibit positive externalities. The value of a unit of the good increases with the expected number of units to be sold. There are two approaches in the analysis of network externalities. The "macro" approach assumes that network externalities exist, and attempts to model their consequences. The "micro" approach attempts to find the cause of network externalities. Network connections bring important effects. Networks established for the purpose of sharing or creating new information provide better, more complete information as more units join and use them. The attractiveness to users of networks increases as they increase in size. The result of these effects is that the self-regulating behavior of demand and price, which is the foundation of traditional microeconomics, no longer applies. Under traditional "negative feedback" economic theory, if demand approaches supply, prices are forced up. This causes demand to decrease and supply to increase, eventually bringing prices back down to their equilibrium level. But information intensive networks are governed by a very different set of "positive feedback" dynamics.

The dynamics of new product adoption is expressed by S-curve (see Figure 2).

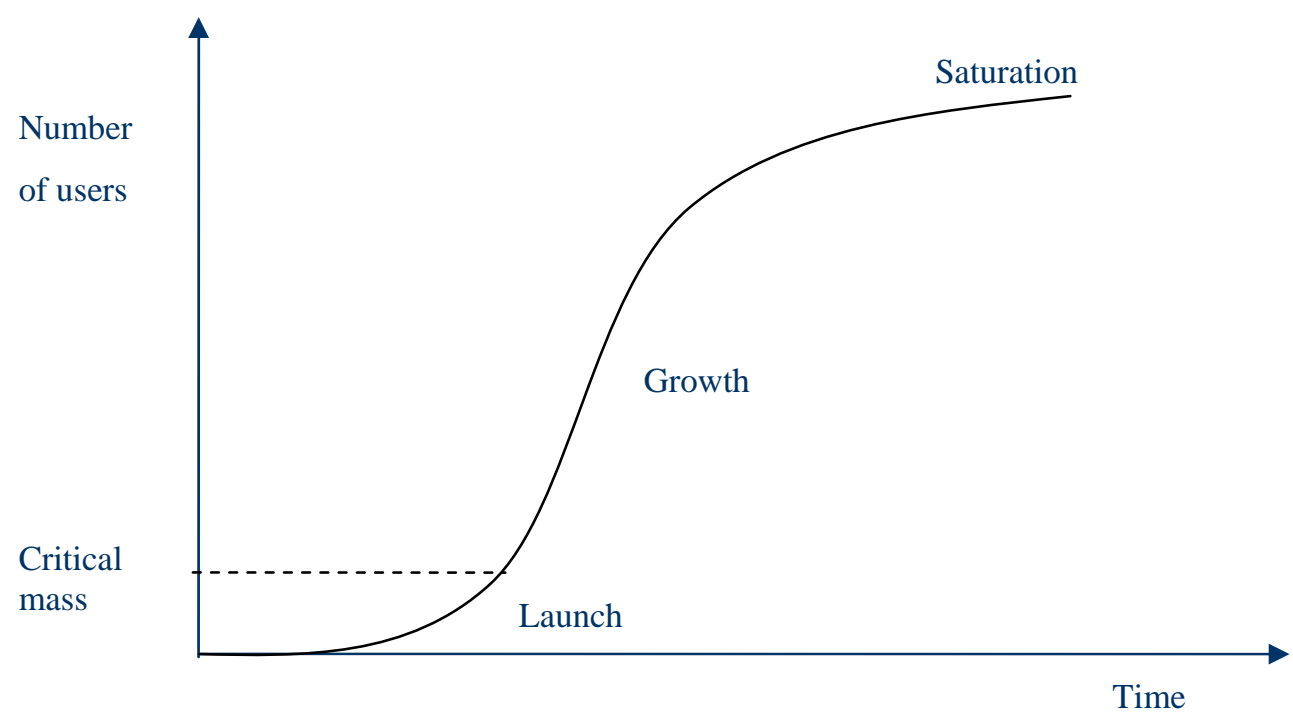

Figure 2 Adaptation dynamics

Then the positive feedback (see Figure 3) can be expressed as: the strong product will be stronger and the weak product will be weaker.

\section{Example}

We use the DHP method for an illustration of positive feedback. The time dependent comparison of two products is expressed by S-curve:

$$
a_{12}(t)=\frac{9}{1+7 \cdot 0,01^{t}} .
$$




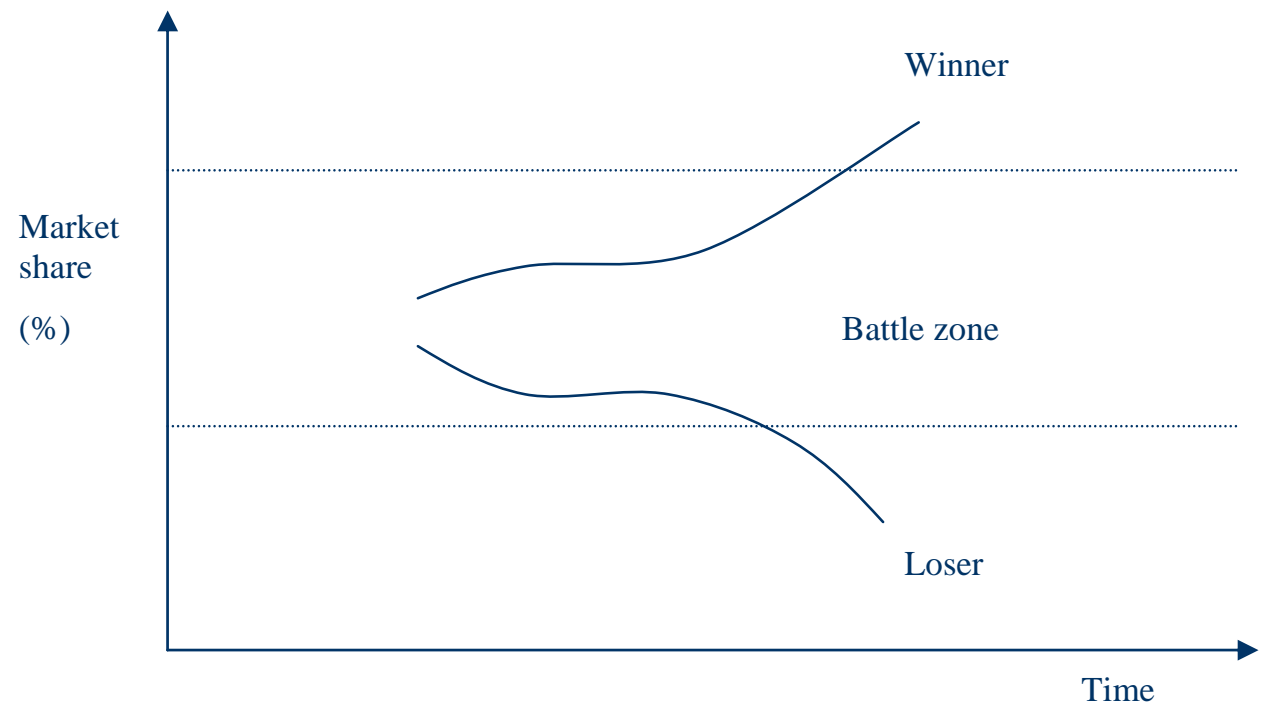

Figure 3 Positive feedback

The paired comparison matrix:

$$
\left[\begin{array}{cc}
1 & a_{12}(t) \\
1 / a_{12}(t) & 1
\end{array}\right]
$$

The numerical data are shown in Table 1 and plotted in Figure 4 and Figure 5.

\begin{tabular}{|c|c|c|c|}
\hline$t$ & $a_{12}(t)$ & $w_{1}(t)$ & $w_{2}(t)$ \\
\hline 0 & 1,13 & 0,53 & 0,47 \\
0,1 & 1,66 & 0,62 & 0,38 \\
0,2 & 2,38 & 0,7 & 0,3 \\
0,3 & 3,26 & 0,77 & 0,23 \\
0,4 & 4,27 & 0,81 & 0,19 \\
0,5 & 5,29 & 0,84 & 0,16 \\
0,6 & 6,24 & 0,86 & 0,14 \\
0,7 & 7,04 & 0,87 & 0,13 \\
0,8 & 7,65 & 0,88 & 0,12 \\
0,9 & 8,10 & 0,89 & 0,11 \\
1 & 8,41 & 0,9 & 0,1 \\
\hline
\end{tabular}

Table 1 Dynamic comparisons 


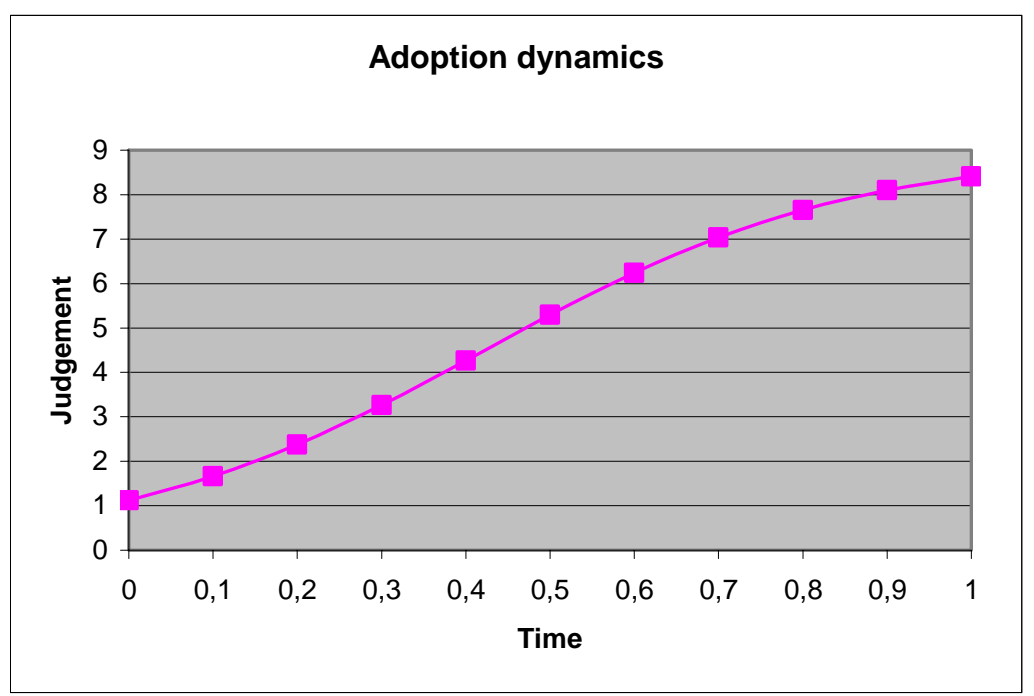

Figure 4 Adaptation dynamics -example

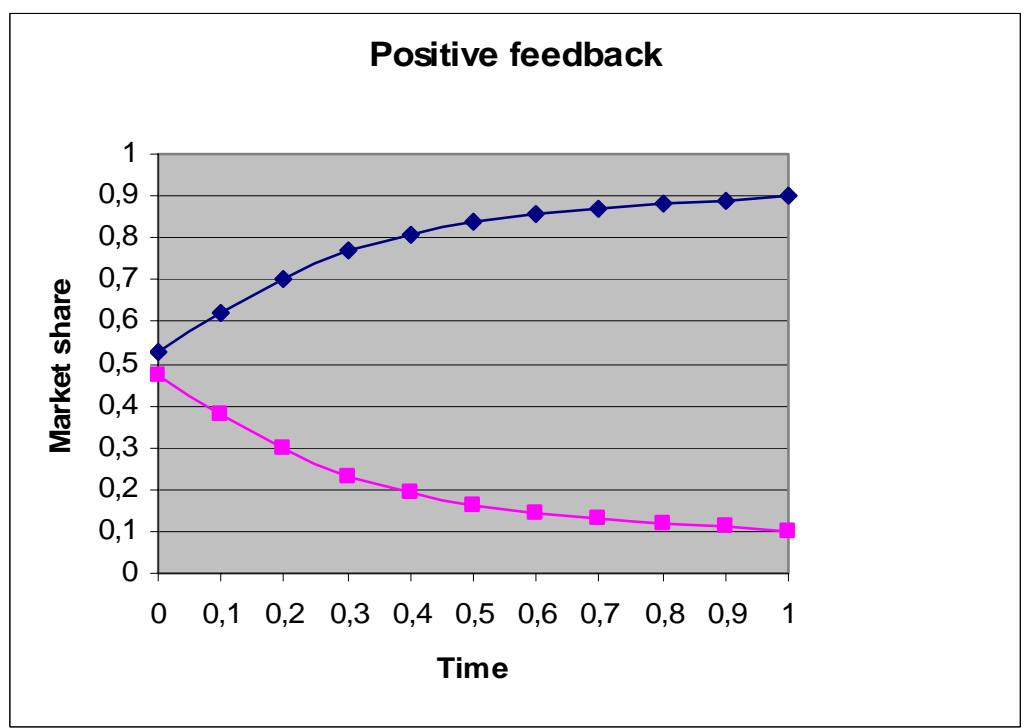

Figure 5 Positive feedback - example

\section{Supplier selection problem}

Supplier selection processes have received considerable attention in business. Sourcing has come up as a very strategic issue in the management of supply chain networks in the modern era of global competition. Sourcing decisions have the capability of impacting the effectiveness of supply chain networks. Determining suitable suppliers in supply chain networks has become a key strategic issue. The nature of these decisions is usually complex and unstructured. Supplier-customer relationships are changing to a cooperative form. The impact of information sharing plays a crucial role. The supplier selection problem is a multiple criteria group decision making problem. Many influence factors such as price, quality, 
flexibility, and delivery performance must be considered to determine suitable suppliers. These influence factors can be divided into quantitative and qualitative factors.

Generally, supplier selection is a multicriteria decision problem. The methods suggested in the related literatures can be classified into two categories:

- mathematical programming models,

- weighting models.

Mathematical programming models are goal programming or multiobjective programming and the linear programming (LP) or mixed integer programming (MIP) with the expression of multiple objectives as constraints. Objective function coefficients should be determined prior to making mathematical programming models. The drawback of goal programming and multiobjective programming is that it requires arbitrary aspiration levels and cannot accommodate subjective criteria. In LP/MIP problem formulations, many of the objectives are regarded as constraints since the LP/MIP formulations allow only one objective function.

The weighting model, which focuses on commonly used evaluation criteria, includes: the linear scoring model and the AHP. The linear scoring model assigns weights and scores arbitrarily. The problem is avoided in the AHP model by converting the priorities into the ratings with regard to each criterion using pair-wise comparisons.

The new features in supplier selection problem are:

- network structure of suppliers and items,

- dynamic connections.

The connections are time dependent. The importance of the criteria and of the suppliers is changed.

In the supplier selection problem are important feedback and dependencies between items or suppliers:

- positive and negative feedback,

- $\quad$ substitution and complementarity

Two items $A$ and $B$ are complementary, if it holds

$$
w(\{A, B\})>w(\{A\})+w(\{B\}) .
$$

Two items $A$ and $B$ are substitute, if it holds

$$
w(\{A, B\})<w(\{A\})+w(\{B\}) .
$$

The Dynamic Network Process seems to be the appropriate method for the specific features of the supplier selection problem in network economy.

\section{Models of network economy}

A model of multi-sector economy focuses on the partitions of the set of firms into clusters induced by subsets formed by firms of the same types. Given the total number of agents, $N$, and the number of possible types, $K$, both of which are assumed to be known and finite, we examine how the $N$-set, that is, the set $\{1,2, \ldots, N\}$ is partitioned into $K$ clusters.

The partition vector $\boldsymbol{v}=\left(v_{1}, v_{2}, \ldots, v_{n}\right)$ is introduced, where $v_{i}$ is the number of clusters with exactly $i$ agents. Then hold the relations 


$$
\sum_{i=1}^{n} v_{i}=k_{n} \leq K \quad, \quad \sum_{i=1}^{n} i v_{i}=n,
$$

where $k_{n}$ is the number of clusters formed by $n$ agents.

For the analysis DNP is used. An alternative approach (Aoki, 2002) is used the master equation (backward Chapman-Kolgomorov equation) as the dynamic equation for the probabilities of state vectors.

In the generalized model we take into account countries, branches, firms and criteria as clusters and different types of connections in the system. There are some dependencies and feedback among elements and clusters also. The whole system is more properly represented as network system. We state some examples of dependencies in the system. There are dependencies among countries given by foreign trade. The branches are interconnected and the flows can be modeled by input-output models. The questionnaire contains questions about networking activities of firms as rates of co-operation with customers and suppliers. The dependencies and feedback should be expressed by appropriate measures. The dynamic version of the model is tested.

We used the alpha version of the ANP software Super Decisions developed by Creative Decisions Foundation (CDF) for some experiments for testing the possibilities of the expression and performance evaluation of the network system (Figure 5).

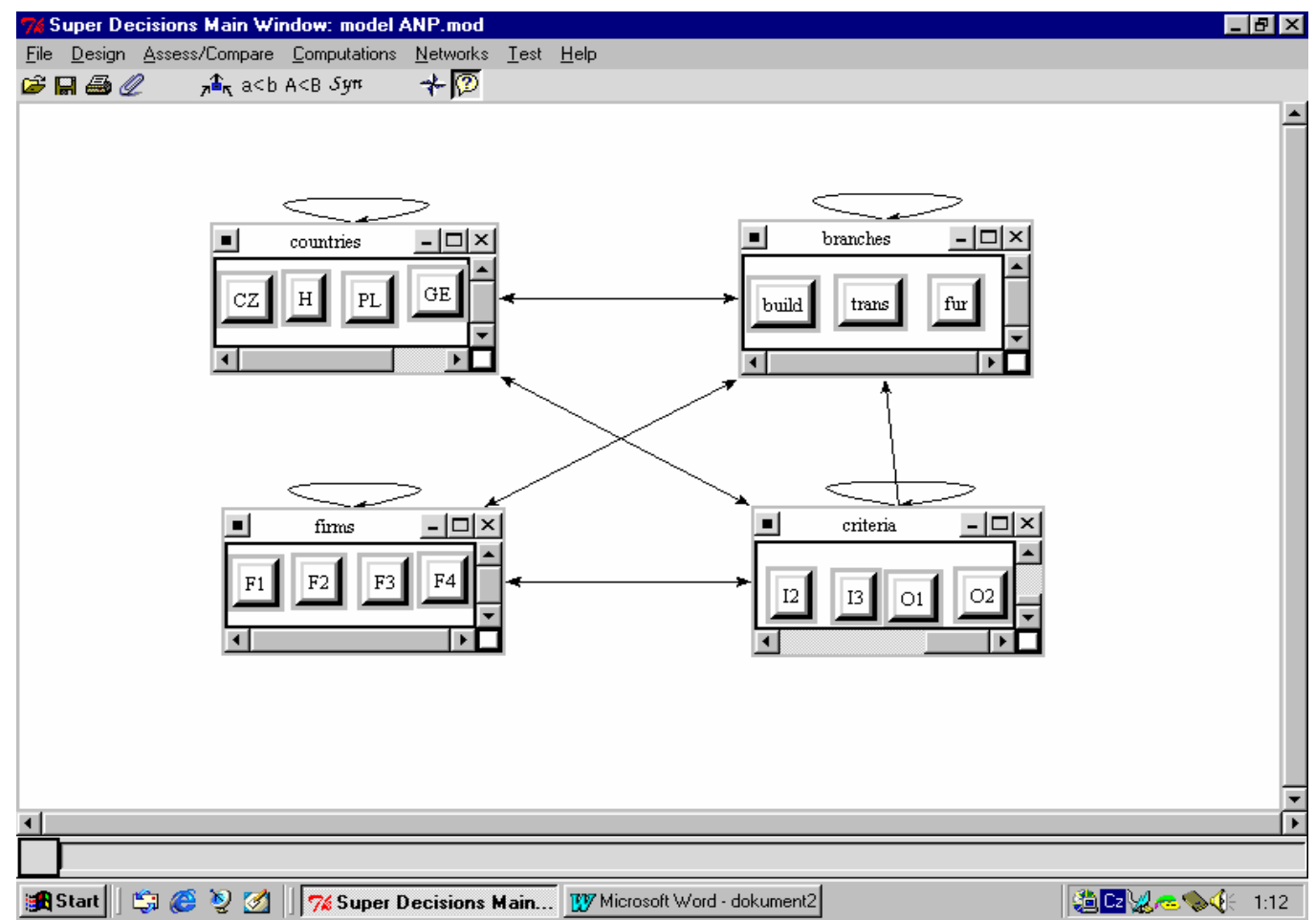

Fig. 6: Generalized DNP model. 


\section{Conclusions}

Analysis and optimization of network economy functioning are challenges for application of modeling approaches. The paper presents models for analyzing network economy. Some specific features of network economy as positive feedback, complementarity, network structure, dynamic environment are analyzed by ANP/DNP methods.

The ANP/DNP approach seems to be an appropriate instrument for analyzing network economy. The specific features are incorporated in a general model. Small example shows a basic principle of the approach but its results cannot be generalized. A large study taking into account a huge number of firms from much many branches is preparing and it will be the aim of our future research.

\section{References}

Aoki M (2002) Aggregate Behavior and Fluctuations in Economics, Cambridge, Cambridge University Press.

CDF (Creative Decisions Foundation) www page (2000)- www.creativedecisions.net.

Economides N (1996) “The Economics of Network,” International Journal of Industrial Organization 14, no.2.

Kelly K (1998) New Rules for the New Economy: 10 Radical Strategies for a Connected World, New York, Viking Press.

Saaty TL (1996) The Analytic Hierarchy Process, Pittsburgh, RWS Publications.

Saaty TL (2001) Decision making with Dependence and Feedback: The Analytic Network Process, Pittsburgh, RWS Publications.

Saaty TL (1999) "Time Dependent Decision-Making; Dynamic Priorities in AHP/ANP: Generalizing from Points to Functions and from Real to Complex Variables," Proceedings of the $7^{\text {th }}$ International Conference on the Analytic Hierarchy Process, Bali, Indonesia, 1-38.

Shapiro C, Varian H (1999) Information Rules: A Strategic Guide to the Network Economy, Boston Harvard Business School Press.

Shy O (2001) The Economics of Network Industries, Cambridge, Cambridge University Press. 